PROCEEDINGS OF THE

AMERICAN MATHEMATICAL SOCIETY

Volume 136, Number 10, October 2008, Pages 3409-3414

S 0002-9939(08)09568-3

Article electronically published on June 5, 2008

\title{
RESIDUALLY REDUCIBLE REPRESENTATIONS OF ALGEBRAS OVER LOCAL ARTINIAN RINGS
}

\author{
JIM BROWN
}

(Communicated by Wen-Ching Winnie Li)

\begin{abstract}
In this paper we generalize a result of Urban on the structure of residually reducible representations on local Artinian rings from the case that the semi-simplification of the residual representation splits into 2 absolutely irreducible representations to the case where it splits into $m \geq 2$ absolutely irreducible representations.
\end{abstract}

\section{Introduction AND STATEMENT OF THE RESUlt}

Let $\mathcal{R}$ be a local Artinian ring, $\mathfrak{m}$ the maximal ideal of $\mathcal{R}$, and $\kappa$ the residue field. For positive integers $m, n$ denote the set of $m$ by $n$ matrices with entries in $\mathcal{R}$ by $\mathrm{M}_{m, n}(\mathcal{R})$ and similarly for those with entries in $\kappa$. If $m=n$, we write $\mathrm{M}_{n}$ for the $n$ by $n$ matrices.

Let $\mathcal{A}$ be an $\mathcal{R}$-algebra. An $n$-dimensional $\mathcal{R}$-representation $\rho$ of $\mathcal{A}$ is an $\mathcal{R}$ algebra homomorphism $\rho: \mathcal{A} \rightarrow \mathrm{M}_{n}(\mathcal{R})$. Equivalently we can write $\rho: \mathcal{A} \rightarrow$ $\operatorname{End}_{\mathcal{R}}(\mathcal{M})$ for $\mathcal{M}$ a free $\mathcal{R}$-module of rank $n$. Given an $\mathcal{R}$-representation $\rho$, we denote the residual representation with values in $\mathrm{M}_{n}(\kappa)$ by $\bar{\rho}$.

In the case where $\bar{\rho}$ is absolutely irreducible, Carayol proved in C94 that $\rho$ is completely determined by its trace. Suppose now that $\bar{\rho}$ is reducible. In the case where the semi-simplification of $\bar{\rho}$ is the sum of two absolutely irreducible representations, Urban gave a generalization of Carayol's result determining the form of $\rho$ in terms of $\rho_{1}$ and $\rho_{2}$; see U99. It was noted in [U99 that the method and result should generalize to the case where the semi-simplification of $\bar{\rho}$ splits into $m$ absolutely irreducible representations. In this paper we state and prove the generalization from $m=2$ to general $m$.

Urban was subsequently able to use a variant of his theorem to produce nontrivial elements in $\operatorname{Ext}_{\mathcal{A}}^{1}\left(\rho_{1}, \rho_{2}\right)$ and used this to give a lower bound on the order of Selmer groups arising from Galois representations attached to classical modular forms ([01]). Our ultimate goal in this line of study is to generalize Urban's result in U01 to include the case where the semi-simplification of the residual representation splits into three absolutely irreducible representations, as in the case when $\rho$ arises as the Galois representation of a cuspidal Siegel eigenform congruent to a Saito-Kurokawa lift. The reader is urged to consult B07 for such a situation.

Received by the editors June 29, 2007.

2000 Mathematics Subject Classification. Primary 16G10.

Key words and phrases. Residually reducible representations, Artinian rings.

(C)2008 American Mathematical Society

Reverts to public domain 28 years from publication 
Let $n_{1}+n_{2}+\cdots+n_{m}$ be a partition of $n$. Let $A \in \mathrm{M}_{n}(\mathcal{R})$. We can write $A$ in blocks as

$$
A=\left(\begin{array}{ccc}
A^{1,1} & \cdots & A^{1, m} \\
\vdots & \ddots & \vdots \\
A^{m, 1} & \cdots & A^{m, m}
\end{array}\right),
$$

where $A^{i, j} \in \mathrm{M}_{n_{i}, n_{j}}(\mathcal{R})$. We denote the $k l^{\text {th }}$ entry of the matrix $A$ by $A_{k, l}$. We denote the matrix in $\mathrm{M}_{n}(\mathcal{R})$ with a 1 in the $i j^{\text {th }}$ entry and 0's elsewhere by $e_{i, j}$. Though we do not include $n$ in our notation for $e_{i, j}$, it should be clear from the context.

The main result of this paper is the following theorem.

Theorem 1.1. Let $\rho, \rho_{1}, \ldots, \rho_{m}$ be $\mathcal{R}$-representations of $\mathcal{A}$. Write $\rho: \mathcal{A} \rightarrow$ $\operatorname{End}_{\mathcal{R}}(\mathcal{M})$ for $\mathcal{M}$ a free $\mathcal{R}$-module of rank $n$. If $\kappa$ has at least $n$ distinct elements and

(1) $\rho$ and $\rho_{1} \oplus \cdots \oplus \rho_{m}$ have the same characteristic polynomials,

(2) $\bar{\rho}_{i}$ is absolutely irreducible for $1 \leq i \leq m$,

(3) $\bar{\rho}_{i} \neq \bar{\rho}_{j}$ for $i \neq j$,

(4) $\bar{\rho}$ is indecomposable and the composition series defining $\bar{\rho}^{\mathrm{ss}}$ is given by

$$
\begin{gathered}
0=\overline{\mathcal{M}}_{0} \subset \overline{\mathcal{M}}_{1} \subset \cdots \subset \overline{\mathcal{M}}_{m-1} \subset \overline{\mathcal{M}}_{m}=\overline{\mathcal{M}}, \\
\text { where } \overline{\mathcal{M}}=\mathcal{M} \otimes_{\mathcal{R}} k \text { and } \overline{\mathcal{M}}_{i} / \overline{\mathcal{M}}_{i-1} \cong \bar{\rho}_{i} \text { for } 1 \leq i \leq m,
\end{gathered}
$$

then there exists $g \in \mathrm{GL}_{n}(\mathcal{R})$ so that

$$
\rho(a)=g\left(\begin{array}{cccc}
\rho_{1}(a) & \star^{1,2} & \cdots & \star^{1, m} \\
0 & \rho_{2}(a) & \cdots & \star^{2, m} \\
\vdots & \ddots & \ddots & \vdots \\
0 & \cdots & 0 & \rho_{m}(a)
\end{array}\right) g^{-1}
$$

for every $a \in \mathcal{A}$.

\section{Proof of the Result}

In this section we provide the proof of Theorem 1.1. We split the proof into several steps and isolate important steps as lemmas. Throughout this section we will write $\rho(a)$ in block form as in equation (11). Thus, anytime an $A^{i, j}(a)$ is used it is referring to the $i j^{\text {th }}$ block in $\rho(a)$. Similarly, the $k l^{\text {th }}$ entry of the matrix $\rho(a)$ is denoted by $A_{k, l}(a)$.

The fact that each $\bar{\rho}_{i}$ is absolutely irreducible implies that $\operatorname{im} \bar{\rho}_{i} \cong \mathrm{M}_{n_{i}}(\kappa)$ (C94). We combine this fact, assumptions (1), (3), and (4) and the BrauerNesbitt theorem to conclude that im $\bar{\rho}^{\mathrm{ss}} \cong \mathrm{M}_{n_{1}}(\kappa) \times \cdots \times \mathrm{M}_{n_{m}}(\kappa)$. Thus, there exists $a_{0} \in \mathcal{A}$ so that the polynomial $\operatorname{det}\left(X-\bar{\rho}\left(a_{0}\right)\right)$ has $n$ distinct $\operatorname{roots} \bar{\alpha}_{1}, \ldots, \bar{\alpha}_{n}$ in $\kappa$ (recall we assumed $\kappa$ has at least $n$ distinct elements). Hensel's lemma guarantees that there exist $n$ distinct elements $\alpha_{1}, \ldots, \alpha_{n}$ that are roots of $\operatorname{det}\left(X-\rho\left(a_{0}\right)\right)$ and $\alpha_{i} \equiv \bar{\alpha}_{i}(\bmod \mathfrak{m})$. Thus, by changing basis if necessary, we may assume

$$
\rho\left(a_{0}\right)=\operatorname{diag}\left(\alpha_{1}, \ldots, \alpha_{n}\right)
$$

and

for $1 \leq i \leq m$.

$$
\rho_{i}\left(a_{0}\right)=\operatorname{diag}\left(\alpha_{n_{i-1}+1}, \ldots, \alpha_{n_{i}}\right)
$$


Lemma 2.1. The $\mathcal{R}$-submodule generated by $\rho\left(a_{0}\right)$ is the set of diagonal matrices $\mathrm{D}_{n}(\mathcal{R}) \subset \mathrm{M}_{n}(\mathcal{R})$.

Proof. Let $\mathcal{B}$ be the $\mathcal{R}$-submodule generated by $\rho\left(a_{0}\right)$. It is clear that $\mathcal{B}$ is contained in $\mathrm{D}_{n}(\mathcal{R})$ as $\rho\left(a_{0}\right)$ is diagonal by construction and $\mathcal{R}$ acts via diagonal scalar matrices. Thus it only remains to show that one in fact obtains all the diagonal matrices in this image.

We will show that $e_{i, i}$ is in $\mathcal{B}$ for $1 \leq i \leq n$. Set

$$
f_{j}=\prod_{i \neq j}\left(\alpha_{i}-\alpha_{j}\right)^{-1}\left(a_{0}-\alpha_{j}\right) .
$$

We use the fact that $\bar{\alpha}_{i} \neq \bar{\alpha}_{j}$ to conclude that $\alpha_{i}-\alpha_{j} \notin \mathfrak{m}$ and since $\mathcal{R}$ is a local ring, we have that $\left(\alpha_{i}-\alpha_{j}\right)^{-1} \in \mathcal{R}$. Thus, we see that $f_{j} \in \mathcal{A}$ for $1 \leq j \leq n$. We have that $\rho\left(f_{j}\right) \in \mathcal{B}$ and $\rho\left(f_{j}\right)=e_{j, j}$, as desired.

Let $a^{\prime} \in \mathcal{A}$ so that $\rho\left(a^{\prime}\right)=\operatorname{diag}\left(\alpha_{1}^{\prime}, \ldots, \alpha_{n}^{\prime}\right)$. Observe that since $\rho\left(a^{\prime}\right)$ is diagonal we have that for any $a \in \mathcal{A}$,

$$
\operatorname{tr}\left(\rho\left(a a^{\prime}\right)\right)=\sum_{i=1}^{m} \operatorname{tr}\left(A^{i, i}(a) \operatorname{diag}\left(\alpha_{n_{i-1}+1}^{\prime}, \ldots, \alpha_{n_{i}}^{\prime}\right)\right) .
$$

We apply assumption (1) to obtain

$$
\operatorname{tr}\left(\rho\left(a a^{\prime}\right)\right)=\sum_{i=1}^{m} \operatorname{tr}\left(\rho_{i}(a) \operatorname{diag}\left(\alpha_{n_{i-1}+1}^{\prime}, \ldots, \alpha_{n_{i}}^{\prime}\right)\right) .
$$

Lemma 2.1 and a judicious choice of $a^{\prime}$ combine with these two equalities to give

$$
\operatorname{tr}\left(A^{i, i}(a)\right)=\operatorname{tr}\left(\rho_{i}(a)\right)
$$

for $1 \leq i \leq m$ and all $a \in \mathcal{A}$. Thus, for all $a_{1}, a_{2} \in \mathcal{A}$ and all $1 \leq i \leq m$,

$$
\begin{aligned}
\operatorname{tr}\left(A^{i, i}\left(a_{1} a_{2}\right)\right) & =\operatorname{tr}\left(\rho_{i}\left(a_{1} a_{2}\right)\right) \\
& =\operatorname{tr}\left(\rho_{i}\left(a_{1}\right) \rho_{i}\left(a_{2}\right)\right) \\
& =\operatorname{tr}\left(\rho_{i}\left(a_{2}\right) \rho_{i}\left(a_{1}\right)\right) \\
& =\operatorname{tr}\left(\rho_{i}\left(a_{2} a_{1}\right)\right) \\
& =\operatorname{tr}\left(A^{i, i}\left(a_{2} a_{1}\right)\right),
\end{aligned}
$$

where we have used that each $\rho_{i}$ is a representation. On the other hand, using that $\rho$ is a representation and so $\rho\left(a_{1} a_{2}\right)=\rho\left(a_{1}\right) \rho\left(a_{2}\right)$ we have

$$
A^{i, i}\left(a_{1} a_{2}\right)=\sum_{j=1}^{m} A^{i, j}\left(a_{1}\right) A^{j, i}\left(a_{2}\right)
$$

and

$$
A^{i, i}\left(a_{2} a_{1}\right)=\sum_{j=1}^{m} A^{i, j}\left(a_{2}\right) A^{j, i}\left(a_{1}\right)
$$

for all $1 \leq i \leq m$. The fact that $\operatorname{tr}\left(A^{i, i}\left(a_{1} a_{2}\right)\right)=\operatorname{tr}\left(A^{i, i}\left(a_{2} a_{1}\right)\right)$ allows us to conclude that

$$
\sum_{\substack{1 \leq j \leq m \\ j \neq i}} \operatorname{tr}\left(A^{i, j}\left(a_{1}\right) A^{j, i}\left(a_{2}\right)\right)=\sum_{\substack{1 \leq j \leq m \\ j \neq i}} \operatorname{tr}\left(A^{i, j}\left(a_{2}\right) A^{j, i}\left(a_{1}\right)\right) .
$$


It is enough to show that for all $1 \leq i<j \leq m, A^{j, i}(a) \in \mathfrak{m}^{k+1} \mathrm{M}_{n_{j}, n_{i}}(\mathcal{R})$ for all $a \in \mathcal{A}$ and all $k \geq 0$. Once we have shown this, the fact that $\mathcal{R}$ is Artinian will give that $A^{j, i}(a)=0$ for all $a \in \mathcal{A}$. We combine this with the result of Carayol mentioned in the introduction and the fact that $\operatorname{tr}\left(A^{i, i}(a)\right)=\operatorname{tr}\left(\rho_{i}(a)\right)$ for all $a \in \mathcal{A}$, $1 \leq i \leq m$ with the fact that $\rho_{i}$ is irreducible to obtain the result.

We proceed by induction on $k$. Note that the case of $k=0$ is given by assumption (41) in Theorem [1.1. Suppose inductively that $A^{j, i}(a) \in \mathfrak{m}^{k} \mathrm{M}_{n_{j}, n_{i}}(\mathcal{R})$ for all $a \in \mathcal{A}$ and all $1 \leq i<j \leq m$. Our first step in proving that $A^{j, i}(a) \in \mathfrak{m}^{k+1} \mathrm{M}_{n_{j}, n_{i}}(\mathcal{R})$ is the following lemma.

Lemma 2.2. Let $a_{1} \in \mathcal{A}$ be in the kernel of $\bar{\rho}$. Under the assumption that $A^{j, i}(a) \in$ $\mathfrak{m}^{k} \mathrm{M}_{n_{j}, n_{i}}(\mathcal{R})$ for all $a \in \mathcal{A}$, one has $A^{j, i}\left(a_{1}\right) \in \mathfrak{m}^{k+1} \mathrm{M}_{n_{j}, n_{i}}(\mathcal{R})$.

Before we can prove this lemma, we need the following result.

Lemma 2.3. Define the subalgebra $T(\kappa)$ of $\mathrm{M}_{n}(\kappa)$ to be the set of block uppertriangular matrices, i.e., matrices of the form

$$
\left(\begin{array}{cccc}
\bar{B}^{1,1} & \bar{B}^{1,2} & \ldots & \bar{B}^{1, m} \\
0 & \bar{B}^{2,2} & \ldots & \bar{B}^{2, m} \\
\vdots & \ddots & \ddots & \vdots \\
0 & \cdots & 0 & \bar{B}^{m, m}
\end{array}\right)
$$

Let $\mathcal{T}(\kappa) \subseteq T(\kappa)$ be the subalgebra consisting of the matrices so that $\bar{B}^{i, j}=0$ if $A^{i, j}(a) \in \mathfrak{m} \mathrm{M}_{n_{i}, n_{j}}(\mathcal{R})$ for all $a \in \mathcal{A}$. The map $\bar{\rho}: \mathcal{A} \rightarrow \mathcal{T}(\kappa)$ is surjective.

Proof. We first observe by assumption (4) of Theorem 1.1 that the image of $\bar{\rho}$ is contained in $\mathcal{T}(\kappa)$. Let $1 \leq i \leq j \leq m$ be such that there exists $a_{i, j} \in \mathcal{A}$ so that $A^{i, j}\left(a_{i, j}\right) \notin \mathfrak{m} \mathrm{M}_{n_{i}, n_{j}}(\mathcal{R})$. Since $\bar{\rho}$ is an algebra map, it is enough to show that for each $u, v$ with $n_{i-1}+1 \leq u \leq n_{i}$ and $n_{j-1}+1 \leq v \leq n_{j}$ there is an element $\gamma \in \mathcal{A}$ so that $\bar{\rho}(\gamma)=e_{u, v} \in \mathrm{M}_{n}(\kappa)$. The fact that $A^{i, j}\left(a_{i, j}\right) \notin \mathfrak{m} \mathrm{M}_{n_{i}, n_{j}}(\mathcal{R})$ gives that there exists $i_{0}, j_{0}$ with $n_{i-1}+1 \leq i_{0} \leq n_{i}$ and $n_{j-1}+1 \leq j_{0} \leq n_{j}$ so that $A_{i_{0}, j_{0}}\left(a_{i, j}\right) \notin \mathfrak{m}$. Let $\alpha \in \mathcal{R}$ such that $\alpha A_{i_{0}, j_{0}}\left(a_{i, j}\right) \equiv 1(\bmod \mathfrak{m})$. Let $f_{u, i_{0}}$ be in $\mathcal{A}$ so that $A^{i, i}\left(f_{u, i_{0}}\right) \equiv e_{u, i_{0}} \in \mathrm{M}_{n_{i}}(\kappa)$. Such a choice is possible by the surjectivity of $\bar{\rho}_{i}$. Note that we are only concerned with the $i i^{\text {th }}$ block here, the entries of the rest of the blocks being irrelevant. Similarly, set $f_{j_{0}, v} \in \mathcal{A}$ so that $A^{j, j}\left(f_{j_{0}, v}\right) \equiv e_{j_{0}, v} \in \mathrm{M}_{n_{j}}(\kappa)$. Define $\gamma=\alpha f_{u, i_{0}} f_{i} a_{i, j} f_{j} f_{j_{0}, v}$ with $f_{i}, f_{j}$ defined as in the proof of Lemma 2.1. Simple matrix multiplication then shows that $\bar{\rho}(\gamma)=e_{u, v}$.

Proof. (of Lemma 2.2) We prove this by appealing to equation (2). The fact that $a_{1} \in \operatorname{ker}(\bar{\rho})$ implies that $A^{i, j}\left(a_{1}\right) \in \mathfrak{m ~ M}_{n_{i}, n_{j}}(\mathcal{R})$. Our induction hypothesis gives that $A^{j, i}\left(a_{2}\right) \in \mathfrak{m}^{k} \mathrm{M}_{n_{j}, n_{i}}(\mathcal{R})$ for all $a_{2} \in \mathcal{A}$ and so we have $A^{i, j}\left(a_{1}\right) A^{j, i}\left(a_{2}\right) \in$ $\mathfrak{m}^{k+1} \mathrm{M}_{n_{i}}(\mathcal{R})$ for all $a_{2} \in \mathcal{A}$. Applying this to equation (2.2) gives that

$$
\sum_{\substack{1 \leq j \leq m \\ j \neq i}} \operatorname{tr}\left(A^{i, j}\left(a_{2}\right) A^{j, i}\left(a_{1}\right)\right) \equiv 0\left(\bmod \mathfrak{m}^{k+1}\right)
$$

for all $a_{2} \in \mathcal{A}$.

Fix a $j_{0}$ with $1 \leq i<j_{0} \leq m$. We restrict to those $a_{2}$ so that $A^{i, j}\left(a_{2}\right) \in$ $\mathfrak{m} \mathrm{M}_{n_{i}, n_{j}}(\mathcal{R})$ if $j \neq j_{0}$. The proof of Lemma 2.3 gives that the restriction to this 
subset of $\mathcal{A}$ still gives a surjective map onto $\mathrm{M}_{n_{i}, n_{j_{0}}}(\kappa)$. For such an $a_{2}$ we have that

$$
\operatorname{tr}\left(A^{i, j_{0}}\left(a_{2}\right) A^{j_{0}, i}\left(a_{1}\right)\right) \equiv 0\left(\bmod \mathfrak{m}^{k+1}\right) .
$$

The surjectivity of the restriction of the map combined with the fact that the trace map is nondegenerate implies that $A^{j_{0}, i}\left(a_{1}\right) \in \mathfrak{m}^{k+1} \mathrm{M}_{n_{j_{0}}, n_{i}}(\mathcal{R})$ as desired. Since $j_{0}$ was arbitrary, we have the result.

We are now able to combine these results to complete the induction and hence our proof of Theorem 1.1. For each $i, j$ with $1 \leq i<j \leq m$ define a map

$$
\begin{aligned}
\Psi_{j, i}: \mathcal{A} & \rightarrow \mathrm{M}_{n_{j}, n_{i}}(\mathcal{R}) \otimes_{\mathcal{R}} \mathfrak{m}^{k} / \mathfrak{m}^{k+1} \\
a & \mapsto A^{j, i}(a)\left(\bmod \mathfrak{m}^{k+1}\right) .
\end{aligned}
$$

Define a map $\Phi_{j, i}: \mathcal{T}(\kappa) \rightarrow \mathrm{M}_{n_{j}, n_{i}}(\mathcal{R}) \otimes_{\mathcal{R}} \mathfrak{m}^{k} / \mathfrak{m}^{k+1}$ as follows. Let $t \in \mathcal{T}(\kappa)$. Lemma 2.3 gives $a \in \mathcal{A}$ so that $\bar{\rho}(a)=t$. Define $\Phi_{j, i}(t)=\Psi_{j, i}(a)$. We need to show that this map is well defined. Suppose there exists $a_{1}, a_{2} \in \mathcal{A}$ so that $\bar{\rho}\left(a_{1}\right)=\bar{\rho}\left(a_{2}\right)$. Then we have $a_{1}-a_{2} \in \operatorname{ker}(\bar{\rho})$. Lemma 2.2 gives that $\Psi_{j, i}\left(a_{1}-a_{2}\right)=0$ and so $\Psi_{j, i}\left(a_{1}\right)=\Psi_{j, i}\left(a_{2}\right)$, and thus our map is well-defined. Note that this gives that the following diagram commutes for each $i, j$ with $1 \leq i<j \leq m$ :

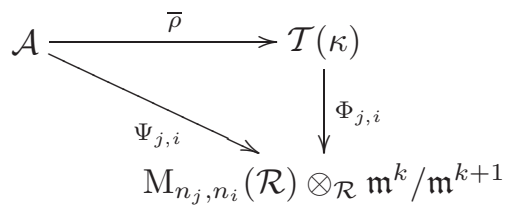

Thus, in order to complete our induction, it is enough to show that the image of $\Phi_{j, i}$ is $\{0\}$ for all $1 \leq i<j \leq m$. The commutativity of the diagram gives that $\Phi_{j, i}$ applied to a diagonal matrix is 0 . Let $\bar{B}$ and $\bar{C}$ be elements of $\mathcal{T}(\kappa)$ with

$$
\bar{B}=\left(\begin{array}{cccc}
\bar{B}^{1,1} & \bar{B}^{1,2} & \ldots & \bar{B}^{1, m} \\
0 & \bar{B}^{2,2} & \ldots & \bar{B}^{2, m} \\
\vdots & \ddots & \ddots & \vdots \\
0 & \ldots & 0 & \bar{B}^{m, m}
\end{array}\right), \quad \bar{C}=\left(\begin{array}{cccc}
\bar{C}^{1,1} & \bar{C}^{1,2} & \ldots & \bar{C}^{1, m} \\
0 & \bar{C}^{2,2} & \ldots & \bar{C}^{2, m} \\
\vdots & \ddots & \ddots & \vdots \\
0 & \ldots & 0 & \bar{C}^{m, m}
\end{array}\right) \text {. }
$$

The surjectivity of $\bar{\rho}$ gives that there exists $b, c \in \mathcal{A}$ so that $\bar{B}^{k, l}=A^{k, l}(b)(\bmod \mathfrak{m})$ and $\bar{C}^{k, l}=A^{k, l}(c)(\bmod \mathfrak{m})$ for all $1 \leq k, l \leq m$. We use the fact that $\Psi_{j, i}(a b)=$ $\sum_{k=1}^{m} A^{j, k}(b) A^{k, i}(c)\left(\bmod \mathfrak{m}^{k+1}\right)$ to conclude that

$$
\Phi_{j, i}(\bar{B} \bar{C})=\sum_{k=1}^{i} \Phi_{j, k}(\bar{B}) \bar{C}^{k, i}+\sum_{k=i+1}^{j} \Phi_{j, k}(\bar{B}) \Phi_{k, i}(\bar{C})+\sum_{k=j+1}^{m} \bar{B}^{j, k} \Phi_{k, i}(\bar{C}) .
$$

To show that the image of $\Phi_{j, i}$ is zero it is enough to show that for each $1 \leq$ $u \leq v \leq m$ we have $\Phi_{j, i}(\bar{C})=0$, where $\bar{C}$ is the matrix defined by $\bar{C}^{i, j}=0$ unless $(i, j)=(u, v)$. Let $\bar{I} \in \mathcal{T}(\kappa)$ be the matrix with $I^{s, t}=0$ if $(s, t) \neq(u, u)$ and $\bar{I}^{u, u}$ 
the $n_{u}$ by $n_{u}$ identity matrix. Then we have $\bar{C}=\bar{I} \bar{C}$ and so

$$
\begin{aligned}
\Phi_{j, i}(\bar{C}) & =\Phi_{j, i}(\bar{I} \bar{C}) \\
& =\sum_{k=1}^{i} \Phi_{j, k}(\bar{I}) \bar{C}^{k, i}+\sum_{k=i+1}^{j} \Phi_{j, k}(\bar{I}) \Phi_{k, i}(\bar{C})+\sum_{k=j+1}^{m} \bar{I}^{j, k} \Phi_{k, i}(\bar{C}) \\
& =\sum_{k=j+1}^{m} \bar{I}^{j, k} \Phi_{k, i}(\bar{C}) \quad\left(\text { since } \Phi_{j, k} \text { of a diagonal matrix is } 0\right) \\
& =0,
\end{aligned}
$$

where the last equality follows from the fact that $\bar{I}^{s, t}=0$ unless $(s, t)=(u, u)$ and in the last sum we have $k>j$, so $(u, u)$ cannot occur as an index on $\bar{I}$. Thus, we have that the image of $\Phi_{j, i}$ is zero for all $1 \leq i<j \leq m$, which concludes the proof of Theorem 1.1.

\section{REFERENCES}

[B07] J. Brown, Saito-Kurokawa lifts and applications to the Bloch-Kato conjecture, Compos. Math. 143, no. 2 (2007), 290-322. MR2309988

[C94] H. Carayol, Formes modulaires et représentations galoisiennes à valeurs dans un anneau local complet, Contemp. Math. 165, Amer. Math. Soc., Providence, RI, 1994, 213-237. MR.1279611 (95i:11059)

[U99] E. Urban, On residually reducible representations on local rings, J. Algebra 212 (1999), 738-742. MR 1676863(2000a:16020)

[U01] E. Urban, Selmer groups and the Eisenstein-Klingen ideal, Duke Math. J. 106, no. 3 (2001), 485-525. MR 1813234(2002b:11073)

Department of Mathematics, California Institute of Technology, Pasadena, CaliFORNIA 91125

Current address: Department of Mathematical Sciences, Clemson University, Clemson, South Carolina 29634-0975

E-mail address: jimlb@caltech.edu 